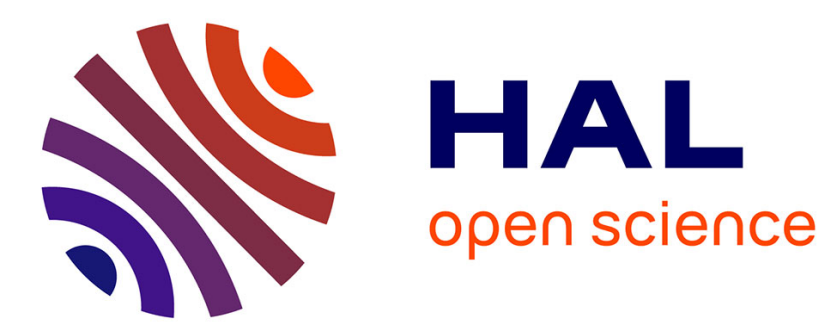

\title{
From the Prékopa-Leindler inequality to modified logarithmic Sobolev inequality \\ Ivan Gentil
}

\section{To cite this version:}

Ivan Gentil. From the Prékopa-Leindler inequality to modified logarithmic Sobolev inequality. Annales de la Faculté des Sciences de Toulouse. Mathématiques., 2008, Sér. 6, 17 (2), pp.291-308. hal$00008521 \mathrm{v} 4$

\section{HAL Id: hal-00008521 \\ https://hal.science/hal-00008521v4}

Submitted on 26 Oct 2007

HAL is a multi-disciplinary open access archive for the deposit and dissemination of scientific research documents, whether they are published or not. The documents may come from teaching and research institutions in France or abroad, or from public or private research centers.
L'archive ouverte pluridisciplinaire HAL, est destinée au dépôt et à la diffusion de documents scientifiques de niveau recherche, publiés ou non, émanant des établissements d'enseignement et de recherche français ou étrangers, des laboratoires publics ou privés. 


\title{
From the Prékopa-Leindler inequality to modified logarithmic Sobolev inequality
}

\author{
Ivan Gentil \\ Ceremade (UMR CNRS no. 7534), Université Paris IX-Dauphine, \\ Place de Lattre de Tassigny, 75775 Paris Cédex 16, France \\ E-mail: gentil@ceremade.dauphine.fr \\ Internet: http://www.ceremade.dauphine.fr/ gentil/
}

26th October 2007

\begin{abstract}
We develop in this paper an improvement of the method given by S. Bobkov and M. Ledoux in BL00. Using the Prékopa-Leindler inequality, we prove a modified logarithmic Sobolev inequality adapted for all measures on $\mathbb{R}^{n}$, with a strictly convex and super-linear potential. This inequality implies modified logarithmic Sobolev inequality, developed in [GGM05, GGM07, for all uniformly strictly convex potential as well as the Euclidean logarithmic Sobolev inequality.
\end{abstract}

\section{Résumé}

Dans cet article nous amélirons la méthode exposée par S. Bobkov et M. Ledoux dans [BL00]. En utilisant l'inégalité de Prékopa-Leindler, nous prouvons une inégalité de Sobolev logarithmique modifiée, adaptée à toutes les mesures sur $\mathbb{R}^{n}$ possédant un potentiel strictement convexe et super-linéaire. Cette inégalité implique en particulier une inégalité de Sobolev logarithmique modifiée, développée dans [GGM05, GGM07, pour les mesures ayant un potentiel uniformément strictement convexe mais aussi une inégalité de Sobolev logarithmique de type euclidien.

\section{Introduction}

The Prékopa-Leindler inequality is the functional form of Brunn-Minkowski inequality. Let $a, b$ be some positive reals such that $a+b=1$, and $u, v, w$ be some non-negative measurable functions on $\mathbb{R}^{n}$. Assume that, for any $x, y \in \mathbb{R}^{n}$, we have

$$
u(x)^{a} v(y)^{b} \leq w(a x+b y),
$$

then

$$
\left(\int u(x) d x\right)^{a}\left(\int v(x) d x\right)^{b} \leq \int w(x) d x
$$

where $d x$ is the Lebesgue measure on $\mathbb{R}^{n}$. If we apply inequality (11) to characteristic functions of bounded measurable sets $A$ and $B$ in $\mathbb{R}^{n}$, we get the multiplicative form of the Brunn-Minkowski inequality

$$
\operatorname{vol}(A)^{a} \operatorname{vol}(B)^{b} \leq \operatorname{vol}(a A+b B),
$$

where $a A+b B=\left\{a x_{A}+b x_{B}, x_{A} \in A, x_{B} \in B\right\}$ and $\operatorname{vol}(A)$ is the Lebesgue measure of the set $A$. One can see for example two interesting reviews on this topic Gup80, Mau04.

Bobkov and Ledoux in [BL00] use the Prékopa-Leindler inequality to prove some functional inequalities like Brascamp-Lieb, Logarithmic Sobolev and Transportation inequalities. 
More precisely, let $\varphi$ be a $\mathcal{C}^{2}$ strictly convex function on $\mathbb{R}^{n}$ and let

$$
d \mu_{\varphi}(x)=e^{-\varphi(x)} d x
$$

be a probability measure on $\mathbb{R}^{n}\left(\int e^{-\varphi(x)} d x=1\right)$. The function $\varphi$ is called the potential of the measure $\mu_{\varphi}$. Bobkov and Ledoux obtained in particular the following two results:

- (Proposition 2.1 of BL00]) Brascamp-Lieb inequality: assume that $\varphi$ is a $\mathcal{C}^{2}$ function on $\mathbb{R}^{n}$, then for all smooth enough functions $g$,

$$
\operatorname{Var}_{\mu_{\varphi}}(g):=\int\left(g-\int g d \mu_{\varphi}\right)^{2} d \mu_{\varphi} \leq \int \nabla g \cdot \operatorname{Hess}(\varphi)^{-1} \nabla g d \mu_{\varphi}
$$

where $\operatorname{Hess}(\varphi)^{-1}$ is the inverse of the Hessian of $\varphi$.

- (Proposition 3.2 of BL00) Assume that for some $c>0$ and $p \geqslant 2$, for all $t, s>0$ with $t+s=1$, and for all $x, y \in \mathbb{R}^{n}, \varphi$ satisfies, as $s$ goes to 0 ,

$$
t \varphi(x)+s \varphi(y)-\varphi(t x+s y) \geqslant \frac{c}{p}(s+o(s))\|x-y\|^{p},
$$

where $\|\cdot\|$ is the Euclidean norm in $\mathbb{R}^{n}$. Then for all smooth enough functions $g$,

$$
\text { Ent }_{\mu_{\varphi}}\left(e^{g}\right):=\int e^{g} \log \frac{e^{g}}{\int e^{g} d \mu_{\varphi}} d \mu_{\varphi} \leq c \int\|\nabla g\|^{q} e^{g} d \mu_{\varphi},
$$

where $1 / p+1 / q=1$. They also give an example: the function $\varphi(x)=\|x\|^{p}+Z_{\varphi}$ (where $Z_{\varphi}$ is a normalization constant) which satisfies inequality (4) for some constant $c>0$.

The main result of this paper is to prove an inequality satisfies for any measure $\mu_{\varphi}$ with a potential strictly convex and super-linear (we also assume a technical hypothesis satisfied by the potential $\varphi)$ ). More precisely we obtain, for all smooth enough functions $g$ on $\mathbb{R}^{n}$,

$$
\mathbf{E n t}_{\mu_{\varphi}}\left(e^{g}\right) \leq \int\left\{x \cdot \nabla g(x)-\varphi^{*}(\nabla \varphi(x))+\varphi^{*}(\nabla \varphi(x)-\nabla g(x))\right\} e^{g(x)} d \mu_{\varphi}(x),
$$

where $\varphi^{*}$ is the Fenchel-Legendre transform of $\varphi, \varphi^{*}(x):=\sup _{z \in \mathbb{R}^{n}}\{x \cdot z-\varphi(z)\}$.

The main application of this result is to extend the modified logarithmic Sobolev inequalities presented in [GGM05, GGM07] for probability measures on $\mathbb{R}$ satisfying a uniform strictly convexity condition. It is well known that if the potential $\varphi$ is $\mathcal{C}^{2}$ on $\mathbb{R}$ such that for all $x \in \mathbb{R}, \varphi^{\prime \prime}(x) \geqslant \lambda>0$, then the measure $\mu_{\varphi}$ defined on (2) verifies the logarithmic Sobolev inequality introduced by Gross in Gro75, for all smooth enough functions $g$, namely

$$
\text { Ent }_{\mu_{\varphi}}\left(e^{g}\right) \leq \frac{1}{2 \lambda} \int g^{\prime 2} e^{g} d \mu_{\varphi}
$$

This result comes from the $\boldsymbol{\Gamma}_{\mathbf{2}}$-criterion of D. Bakry and M. Émery, see BE85 or $\mathrm{ABC}^{+} 00$ for a review. We then improve the classical logarithmic Sobolev inequality of Gross, in the situation where if the potential is even with $\varphi(0)=0$ and satisfies

$$
\forall x \in \mathbb{R}, \quad \varphi^{\prime \prime}(x) \geqslant \lambda>0 \text { and } \lim _{|x| \rightarrow \infty} \varphi^{\prime \prime}(x)=\infty .
$$

Adding a technical hypothesis (see Section 3.1), we show that for all smooth functions $g$,

$$
\operatorname{Ent}_{\mu_{\varphi}}\left(e^{g}\right) \leq \int H_{\varphi}\left(g^{\prime}\right) e^{g} d \mu_{\varphi}
$$


where

$$
H_{\varphi}(x)=\left\{\begin{array}{rrr}
C^{\prime} \varphi^{*}\left(\frac{x}{2}\right), & \text { if } & |x|>C \\
\frac{1}{2 \lambda} x^{2}, & \text { if } & |x| \leq C
\end{array}\right.
$$

for some constants $C, C^{\prime}, \lambda>0$ depending on $\varphi$. Remark that we always have

$$
\forall x \in \mathbb{R}, \quad H_{\varphi}(x) \leq C^{\prime \prime} x^{2},
$$

for some other constant $C^{\prime \prime}$. This inequality implies concentration inequalities which are more adapted to the measure studied, as we will see in Section 3.1.

The next section is divided into two subsections. In the first one we state the main theorem of this article, inequality (6). In the second subsection, we explain how this result improves results of BL00]. In particular, inequality (5) or Brascamp-Lieb inequality (3). Section 3 deals with some applications. The first one is an improvement of the a classical consequence of the $\boldsymbol{\Gamma}_{\mathbf{2}}$-criterion of Bakry-Émery for measures on $\mathbb{R}$. We obtain then a global view of modified logarithmic Sobolev inequality for log-concave measures as introduced in joint work with A. Guillin and L. Miclo in [GGM05, GGM07]. Finally, we explain how the main theorem is equivalent to the Euclidean logarithmic Sobolev inequality. As a consequence, a short proof of the generalization given in [DPD03, Gen03, AGK04 is obtained.

\section{Inequality for log-concave measures}

\subsection{The main theorem}

Theorem 2.1 Let $\varphi$ be a $\mathcal{C}^{2}$ strictly convex function on $\mathbb{R}^{n}$, such that

$$
\lim _{\|x\| \rightarrow \infty} \frac{\varphi(x)}{\|x\|}=\infty
$$

Denotes by $\mu_{\varphi}(d x)=e^{-\varphi(x)} d x$ a probability measure on $\mathbb{R}^{n}$, where $d x$ is the Lebesgue measure on $\mathbb{R}^{n},\left(\int e^{-\varphi(x)} d x=1\right)$. Assume that $\mu_{\varphi}$ satisfies for any $R>0$,

$$
\int\left(\|z\|+\left\|y_{0}\right\|+R\right)^{2}\left(\|\nabla \varphi(z)\|+\sup _{y ;\left\|y-z+y_{0}\right\| \leq R}\|\operatorname{Hess}(\varphi)(y)\|\right) d \mu_{\varphi}(z)<+\infty,
$$

where $y_{0}$ satisfies $\left\|\nabla \varphi\left(y_{0}\right)\right\| \leq\|\nabla \varphi(z)\|+R$.

If $\varphi^{*}$ is the Fenchel-Legendre transform of $\varphi, \varphi^{*}(x):=\sup _{z \in \mathbb{R}^{n}}\{x \cdot z-\varphi(z)\}$, then for all smooth enough functions $g$ on $\mathbb{R}^{n}$, one gets

$$
\text { Ent }_{\mu_{\varphi}}\left(e^{g}\right) \leq \int\left\{x \cdot \nabla g(x)-\varphi^{*}(\nabla \varphi(x))+\varphi^{*}(\nabla \varphi(x)-\nabla g(x))\right\} e^{g(x)} d \mu_{\varphi}(x) .
$$

Lemma 2.2 Let $\varphi$ satisfying conditions on Theorem 2.1 then we have

- $\nabla \varphi$ is a bijection on $\mathbb{R}^{n}$ to $\mathbb{R}^{n}$

- $\lim _{\|x\| \rightarrow \infty} \frac{x \cdot \nabla \varphi(x)}{\|x\|}=+\infty$.

\section{Proof}

$\triangleleft$ Condition (7) implies that for all $x \in \mathbb{R}^{n}$ the supremum of $x \cdot z-\varphi(z)$ for $y \in \mathbb{R}^{n}$ is reached for some $y \in \mathbb{R}^{n}$. Then $y$ satisfies $x=\nabla \varphi(y)$ and it proves that $\nabla \varphi$ is a surjection. Then the strict convexity of $\varphi$ implies that $\nabla \varphi$ is a bijection.

The function $\varphi$ is convex then for all $x \in \mathbb{R}^{n}, x \cdot \nabla \varphi(x) \geqslant \varphi(x)-\varphi(0)$, (7) implies the second properties satisfied by $\varphi$. $\triangleright$

The proof of the theorem is based on the following lemma: 
Lemma 2.3 Let $g$ be a $\mathcal{C}^{\infty}$ function with a compact support on $\mathbb{R}^{n}$. Let $s, t \geqslant 0$ with $t+s=1$ and denotes

$$
\forall z \in \mathbb{R}^{n}, \quad g_{s}(z)=\sup _{z=t x+s y}(g(x)-(t \varphi(x)+s \varphi(y)-\varphi(t x+s y))) .
$$

Then there exists $R \geqslant 0$ such that, when $s$ goes to 0 ,

$$
\begin{aligned}
g_{s}(z)=g(z)+ & s\left\{z \cdot \nabla g(z)-\varphi^{*}(\nabla \varphi(z))+\varphi^{*}(\nabla \varphi(x)-\nabla g(x))\right\} \\
& +\left(\left(\|z\|+\left\|y_{0}\right\|+R\right)\|\nabla \varphi(z)\|+\left(\|z\|+\left\|y_{0}\right\|+R\right)^{2} \sup _{y ;\left\|y-z+y_{0}\right\| \leq R}\|\operatorname{Hess}(\varphi)(y)\|\right) O\left(s^{2}\right),
\end{aligned}
$$

where $y_{0}$ satisfies $\left\|\nabla \varphi\left(y_{0}\right)\right\| \leq\|\nabla \varphi(z)\|+R$ and $O\left(s^{2}\right)$ is uniform on $z \in \mathbb{R}^{n}$.

\section{Proof}

$\triangleleft$ Let $s \in] 0,1 / 2[$ and $x=z / t-(s / t) y$, hence

$$
g_{s}(z)=\varphi(z)+\sup _{y \in \mathbb{R}^{n}}\left(g\left(\frac{z}{t}-\frac{s}{t} y\right)-t \varphi\left(\frac{z}{t}-\frac{s}{t} y\right)-s \varphi(y)\right) .
$$

Due to the fact that $g$ has a compact support and by property (đ) there exists $y_{s} \in \mathbb{R}^{n}$ such that

$$
\sup _{y \in \mathbb{R}^{n}}\left(g\left(\frac{z}{t}-\frac{s}{t} y\right)-t \varphi\left(\frac{z}{t}-\frac{s}{t} y\right)-s \varphi(y)\right)=g\left(\frac{z}{t}-\frac{s}{t} y_{s}\right)-t \varphi\left(\frac{z}{t}-\frac{s}{t} y_{s}\right)-s \varphi\left(y_{s}\right) .
$$

Moreover, $y_{s}$ satisfies

$$
\nabla g\left(\frac{z}{t}-\frac{s}{t} y_{s}\right)-t \nabla \varphi\left(\frac{z}{t}-\frac{s}{t} y_{s}\right)+t \nabla \varphi\left(y_{s}\right)=0
$$

Lemma 2.2 implies that there exists a unique solution $y_{0}$ of the equation

$$
\nabla \varphi\left(y_{0}\right)=\nabla \varphi(z)-\nabla g(z), \quad y_{0}=(\nabla \varphi)^{-1}(\nabla \varphi(z)-\nabla g(z))
$$

We prove now that $\lim _{s \rightarrow 0} y_{s}=y_{0}$.

First we show that there exists $A \geqslant 0$ such that $\forall s \in] 0,1 / 2\left[,\left\|y_{s}\right\| \leq A\right.$. Indeed, if the function $y_{s}$ is not bounded one can found $\left(s_{k}\right)_{k \in \mathbb{N}}$ such that $s_{k} \rightarrow 0$ and $\left\|y_{s_{k}}\right\| \rightarrow \infty$. Definition of $y_{s}$ implies that

$$
g\left(\frac{z}{t}-\frac{s}{t} y_{s}\right)-t \varphi\left(\frac{z}{t}-\frac{s}{t} y_{s}\right)-s \varphi\left(y_{s}\right) \geqslant g\left(\frac{z}{t}\right)-t \varphi\left(\frac{z}{t}\right)
$$

Due to the fact that $\lim _{\|x\| \rightarrow \infty} \varphi(x)=\infty$ and since $g$ is bounded we obtain $s_{k} y_{s_{k}}=O(1)$. Next using (10) one get

$$
\frac{y_{s} \cdot \nabla g\left(\frac{z}{t}-\frac{s}{t} y_{s}\right)}{\left\|y_{s}\right\|}-t \frac{y_{s} \cdot \nabla \varphi\left(\frac{z}{t}-\frac{s}{t} y_{s}\right)}{\left\|y_{s}\right\|}+t \frac{y_{s} \cdot \nabla \varphi\left(y_{s}\right)}{\left\|y_{s}\right\|}=0 .
$$

The last equality is an contradiction with the second assertion of Lemma 2.2 which prove that the

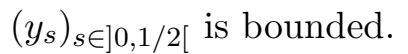

Let $\hat{y}$ an accumulation point of the function $y_{s}$, when $s$ tends to 0 . Then $\hat{y}$ satisfies equation (11). By unicity of the solution of (11) we get $\hat{y}=y_{0}$. Therefore we have proved that $\lim _{s \rightarrow 0} y_{s}=y_{0}$.

Taylor formula gives

$$
\varphi\left(\frac{z}{t}-\frac{s}{t} y_{s}\right)=\varphi(z)+s\left(\frac{z}{t}-\frac{y_{s}}{t}\right) \cdot \nabla \varphi(z)+s^{2} \int_{0}^{1}(1-t)\left(\frac{z}{t}-\frac{y_{s}}{t}\right) \cdot \operatorname{Hess}(\varphi)\left(\frac{z}{t}-s \frac{y_{s}}{t}\right)\left(\frac{z}{t}-\frac{y_{s}}{t}\right) d t
$$


and the same for $g$. Using the continuity of $y_{s}$ at $s=0$, ones gets

$$
\begin{aligned}
\varphi\left(\frac{z}{t}-\frac{s}{t} y_{s}\right)=\varphi(z) & +s\left(z-y_{0}\right) \cdot \nabla \varphi(z) \\
+ & \left(\left(z-y_{0}\right) \cdot \nabla \varphi(z)+\sup _{t \in[0,1 / 2]}\left\|\frac{z}{t}-\frac{y_{t}}{t}\right\|^{2} \sup _{t \in[0,1 / 2]}\left\|\operatorname{Hess}(\varphi)\left(\frac{z}{t}-\frac{y_{s}}{t}\right)\right\|\right) O\left(s^{2}\right) .
\end{aligned}
$$

and the same for $g$

$$
\begin{aligned}
g\left(\frac{z}{t}-\frac{s}{t} y_{s}\right)=g(z)+ & s\left(z-y_{0}\right) \cdot \nabla g(z)+ \\
& \left(\left(z-y_{0}\right) \cdot \nabla g(z)+\sup _{t \in[0,1 / 2]}\left\|\frac{z}{t}-\frac{y_{t}}{t}\right\|^{2} \sup _{t \in[0,1 / 2]}\left\|\operatorname{Hess}(g)\left(\frac{z}{t}-\frac{y_{s}}{t}\right)\right\|\right) O\left(s^{2}\right) .
\end{aligned}
$$

As a consequence,

$$
\begin{aligned}
& g_{s}(z)=g(z)+s\left\{\varphi(z)-\varphi\left(y_{0}\right)+\left(z-y_{0}\right) \cdot(\nabla g(z)-\nabla \varphi(z))\right\} \\
& \quad+\left(\left(z-y_{0}\right) \cdot(\nabla g(z)-\nabla \varphi(z))+\sup _{t \in[0,1 / 2]}\left\|\frac{z}{t}-\frac{y_{t}}{t}\right\|^{2} \sup _{t \in[0,1 / 2]}\left\|\operatorname{Hess}(\varphi+g)\left(\frac{z}{t}-\frac{y_{s}}{t}\right)\right\|\right) O\left(s^{2}\right) .
\end{aligned}
$$

The function $g$ is $\mathcal{C}^{\infty}$ with a compact support then one obtains using (11) and the expression of the Fenchel-Legendre transformation for a strictly convex function

$$
\forall x \in \mathbb{R}^{n}, \quad \varphi^{*}(\nabla \varphi(z))=\nabla \varphi(z) \cdot z-\varphi(z)
$$

we get the result. $\triangleright$

We are now ready to deduce our main result:

Proof of Theorem 2.1

$\triangleleft$ The proof is based on the proof of Theorem 3.2 of [BL00]. First we prove inequality (9) for all functions $g, \mathcal{C}^{\infty}$ with compact support on $\mathbb{R}^{n}$.

Let $t, s \geqslant 0$ with $t+s=1$ and denote for $z \in \mathbb{R}^{n}$,

$$
g_{t}(z)=\sup _{z=t x+s y}(g(x)-(t \varphi(x)+s \varphi(y)-\varphi(t x+s y))) .
$$

We apply Prékopa-Leindler inequality to the functions

$$
u(x)=\exp \left(\frac{g(x)}{t}-\varphi(x)\right), \quad v(y)=\exp (-\varphi(y)), \quad w(z)=\exp \left(g_{s}(z)-\varphi(z)\right),
$$

to get

$$
\left(\int \exp (g / t) d \mu_{\varphi}\right)^{t} \leq \int \exp \left(g_{s}\right) d \mu_{\varphi}
$$

The differentiation of the $L^{p}$ norm gives the entropy, and thanks to a Taylor's formula we get

$$
\left(\int \exp (g / t) d \mu_{\varphi}\right)^{t}=\int e^{g} d \mu_{\varphi}+s \mathbf{E n t}_{\mu_{\varphi}}\left(e^{g}\right)+O\left(s^{2}\right) .
$$

Then applying Lemma 2.3 and inequality (8) yield

$$
\begin{aligned}
& \int \exp \left(g_{s}\right) d \mu_{\varphi}= \\
& \qquad e^{g} \mu_{\varphi}+s \int\left\{z \cdot \nabla g(z)-\varphi^{*}(\nabla \varphi(z))+\varphi^{*}(\nabla \varphi(z)-\nabla g(z))\right\} e^{g(z)} d \mu_{\varphi}(z)+O\left(s^{2}\right) .
\end{aligned}
$$


When $s$ goes to 0 , inequality (9) arises and can be extended for all smooth enough functions $g . \triangleright$

Note that hypothesis (8) is satisfied by a large class of convex functions. For example if $\varphi(x)=$ $\|x\|^{2} / 2+(n / 2) \log (2 \pi)$ we obtain the classical logarithmic Sobolev of Gross for the canonical Gaussian measure on $\mathbb{R}^{n}$, with the optimal constant.

\subsection{Remarks and examples}

In the next corollary we recall a classical result of perturbation. If $\Phi$ is a function on $\mathbb{R}^{n}$ such that $\int e^{-\Phi} d x<\infty$ we note the probability measure $\mu_{\Phi}$ by

$$
d \mu_{\Phi}(x)=\frac{e^{-\Phi(x)}}{Z_{\Phi}} d x
$$

where $Z_{\Phi}=\int e^{-\Phi(x)} d x$.

Corollary 2.4 Assume that $\varphi$ satisfies conditions of Theorem 2.1. Let $\Phi=\varphi+U$, where $U$ is a bounded function on $\mathbb{R}^{n}$ and denote by $\mu_{\Phi}$ the measure defined by (12).

Then for all smooth enough functions $g$ on $\mathbb{R}^{n}$, one has

$$
\operatorname{Ent}_{\mu_{\Phi}}\left(e^{g}\right) \leq e^{2 o s c(U)} \int\left\{x \cdot \nabla g(x)-\varphi^{*}(\nabla \varphi(x))+\varphi^{*}(\nabla \varphi(x)-\nabla g(x))\right\} e^{g(x)} d \mu_{\Phi}(x),
$$

where osc $(U)=\sup (U)-\inf (U)$.

\section{Proof}

$\triangleleft$ First we observe that

$$
e^{-\operatorname{osc}(U)} \leq \frac{d \mu_{\Phi}}{d \mu_{\varphi}} \leq e^{\operatorname{osc}(U)}
$$

Moreover we have for all probability measures $\nu$ on $\mathbb{R}^{n}$,

$$
\operatorname{Ent}_{\nu}\left(e^{g}\right)=\inf _{a \geqslant 0}\left\{\int\left(e^{g} \log \frac{e^{g}}{a}-e^{g}+a\right) d \nu\right\} .
$$

Using the fact that for all $x, a>0, x \log \frac{x}{a}-x+a \geqslant 0$, we get

$$
e^{-\mathrm{osc}(U)} \mathbf{E n t}_{\mu_{\Phi}}\left(e^{g}\right) \leq \mathbf{E n t}_{\mu_{\varphi}}\left(e^{g}\right) \leq e^{\mathrm{osc}(U)} \mathbf{E n t}_{\mu_{\Phi}}\left(e^{g}\right) .
$$

Then if $g$ a smooth enough function on $\mathbb{R}^{n}$ we have

$$
\begin{aligned}
\text { Ent }_{\mu_{\Phi}}\left(e^{g}\right) & \leq e^{\operatorname{osc}(U)} \mathbf{E n t}_{\mu_{\varphi}}\left(e^{g}\right) \\
& \leq e^{\operatorname{osc}(U)} \int\left\{x \cdot \nabla g(x)-\varphi^{*}(\nabla \varphi(x))+\varphi^{*}(\nabla \varphi(x)-\nabla g(x))\right\} e^{g(x)} d \mu_{\varphi}(x) .
\end{aligned}
$$

The convexity of $\varphi^{*} \mathbb{R}^{n}$ and th relation $\nabla \varphi^{*}(\nabla \varphi(x))=x$ lead to

$$
\forall x \in \mathbb{R}^{n}, \quad x \cdot \nabla g(x)-\varphi^{*}(\nabla \varphi(x))+\varphi^{*}(\nabla \varphi(x)-\nabla g(x)) \geqslant 0 .
$$

Finally by (14) we get

$$
\text { Ent }_{\mu_{\Phi}}\left(e^{g}\right) \leq e^{2 \operatorname{osc}(U)} \int\left\{x \cdot \nabla g(x)-\varphi^{*}(\nabla \varphi(x))+\varphi^{*}(\nabla \varphi(x)-\nabla g(x))\right\} e^{g} d \mu_{\Phi} .
$$

$\triangleright$ 
Remark 2.5 It is not necessary to state a tensorization result, as we may obtain exactly the same expression when computing directly with a product measure.

Theorem 2.1 implies also examples given in BL00] and [BZ05].

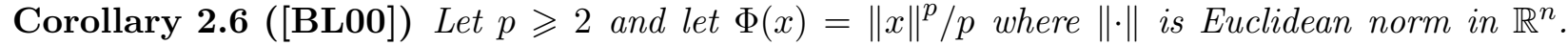
Then there exists $c>0$, such that for all smooth enough functions $g$,

$$
\text { Ent }_{\mu_{\Phi}}\left(e^{g}\right) \leq c \int\|\nabla g\|^{q} e^{g} d \mu_{\Phi},
$$

where $1 / p+1 / q=1$ and $\mu_{\Phi}$ is defined on (12).

\section{Proof}

$\triangleleft$ Using Theorem 2.1, we just have to prove that there exists $c>0$ such that,

$$
\forall x, y \in \mathbb{R}^{n}, \quad x \cdot y-\Phi^{*}(\nabla \Phi(x))+\Phi^{*}(\nabla \Phi(x)-y) \leq c\|y\|^{q} .
$$

Assume that $y \neq 0$ and define the function $\psi$ by,

$$
\psi(x, y)=\frac{x \cdot y-\Phi^{*}(\nabla \Phi(x))+\Phi^{*}(\nabla \Phi(x)-y)}{\|y\|^{q}} .
$$

Then $\psi$ is a bounded function. We know that $\Phi^{*}(x)=\|x\|^{q} / q$. Choosing $z=x\|x\|^{p-2} /\|y\|$ and denoting $e=y /\|y\|$, we obtain

$$
\psi(x, y)=\bar{\psi}(z, e)=z \cdot e\|z\|^{q-2}-\frac{1}{q}\|z\|^{q}+\frac{1}{q}\|z-e\|^{q} .
$$

Taylor's formula then yields $\bar{\psi}(z, e)=O\left(\|z\|^{q-2}\right)$. But $p \geqslant 2$ implies that $q \leq 2$, so that $\bar{\psi}$ is a bounded function. We then get the result with $c=\sup \bar{\psi}=\sup \psi$. $\triangleright$

Optimal transportation is also used by Cordero-Erausquin, Gangbo and Houdré in [CEGH04 to prove the particular case of the inequality (15).

In Proposition 2.1 of [BL00], Bobkov and Ledoux prove that the Prékopa-Leindler inequality implies Brascamp-Lieb inequality. In our case, Theorem 2.1 also implies Brascamp-Lieb inequality, as we can see in the next corollary.

Corollary 2.7 Let $\varphi$ satisfying conditions of Theorem 2.1. Then for all smooth enough functions $g$ we get,

$$
\operatorname{Var}_{\mu_{\varphi}}(g) \leq \int \nabla g \cdot \operatorname{Hess}(\varphi)^{-1} \nabla g d \mu_{\varphi}
$$

where Hess $(\varphi)^{-1}$ denote the inverse of the Hessian of $\varphi$.

\section{Proof}

$\triangleleft$ Assume that $g$ is a $\mathcal{C}^{\infty}$ function with a compact support and apply inequality (9) with the function $\epsilon g$ where $\epsilon>0$. Taylor's formula gives

$$
\mathbf{E n t}_{\mu_{\varphi}}(\exp \epsilon g)=\frac{\epsilon^{2}}{2} \operatorname{Var}_{\mu_{\varphi}}(g)+o\left(\epsilon^{2}\right)
$$

and

$$
\begin{gathered}
\int\left\{x \cdot \nabla g(x)-\varphi^{*}(\nabla \varphi(x))+\varphi^{*}(\nabla \varphi(x)-\nabla g(x))\right\} e^{g}(x) d \mu_{\varphi}(x)= \\
\int \frac{\epsilon^{2}}{2} \nabla g \cdot \operatorname{Hess}\left(\varphi^{*}\right)(\nabla \varphi) \nabla g d \mu_{\varphi}+o\left(\epsilon^{2}\right) .
\end{gathered}
$$

Because of $\nabla \varphi^{*}(\nabla \varphi(x))=x$, one has $\operatorname{Hess}\left(\varphi^{*}\right)(\nabla \varphi)=\operatorname{Hess}(\varphi)^{-1}$ which finished the proof. $\quad$ 
Remark 2.8 Let $\varphi$ satisfying the conditions of Theorem 2.1, and $L$ be defined by

$$
\forall x, y \in \mathbb{R}^{n}, \quad L(x, y)=\varphi(y)-\varphi(x)+(y-x) \cdot \nabla \varphi(x) .
$$

The convexity of $\varphi$ implies that $L(x, y) \geqslant 0$ for all $x, y \in \mathbb{R}^{n}$. Let $F$ be a density of probability with respect to the measure $\mu_{\varphi}$, we defined the following Wasserstein distance with the cost function $L$ by

$$
W_{L}\left(F d \mu_{\varphi}, d \mu_{\varphi}\right)=\inf \left\{\int L(x, y) d \pi(x, y)\right\},
$$

where the infimum is taken over all probability measures $\pi$ on $\mathbb{R}^{n} \times \mathbb{R}^{n}$ with marginal distributions $F d \mu_{\varphi}$ and $d \mu_{\varphi}$. Bobkov and Ledoux proved in BLOd the following transportation inequality

$$
W_{L}\left(F d \mu_{\varphi}, d \mu_{\varphi}\right) \leq \mathbf{E n t}_{\mu_{\varphi}}(F) .
$$

The main result of Otto and Villani in OVOd is the following: Classical logarithmic Sobolev inequality of Gross (when $\varphi(x)=\|x\|^{2} / 2+(n / 2) \log (2 \pi)$ ) implies the transportation inequality (16) for all functions $F$, density of probability with respect to $\mu_{\varphi}$ (see also BGL01] for an another proof). The method developed in [BGL01], enables to extend the property for $\varphi(x)=\|x\|^{p}+Z_{\varphi}(p \geqslant 2)$. In the general case, inequality proved in this article, we do not know if the modified logarithmic Sobolev inequality (9) implies transportation inequality (16).

\section{Applications}

\subsection{Application to modified logarithmic Sobolev inequalities}

In GGM05, GGM07, a modified logarithmic Sobolev inequality for measure $\mu_{\varphi}$ on $\mathbb{R}$ is given with a potential between $|x|$ and $x^{2}$. More precisely let $\Phi$ be a function on the real line and assume that $\Phi$ is even and satisfies the following property: there exist $M \geqslant 0$ and $0<\varepsilon \leq 1 / 2$ such that,

$$
\forall x \geqslant M, \quad(1+\varepsilon) \Phi(x) \leq x \Phi^{\prime}(x) \leq(2-\varepsilon) \Phi(x) .
$$

Then there exist $A, B, D>0$ such that for all smooth functions $g$ we have

$$
\operatorname{Ent}_{\mu_{\Phi}}\left(e^{g}\right) \leq A \int H_{\Phi}\left(g^{\prime}\right) e^{g} d \mu_{\Phi}
$$

where

$$
H_{\Phi}(x)=\left\{\begin{aligned}
\Phi^{*}(B x) & \text { if }|x| \geqslant D \\
x^{2} & \text { if }|x| \leq D
\end{aligned}\right.
$$

and $\mu_{\Phi}$ is defined on (12).

The proof of inequality (17) is rather technical and is divided in two parts: the large and the small entropy. Using Theorem 2.1 one obtains two results in this direction. In the next theorem, we extend (17) in the case where the potential is "bigger" than $x^{2}$.

Theorem 3.1 Let $\varphi$ be a real function satisfying conditions of Theorem 2.1. Assume that $\varphi$ is even, $\varphi(0)=0, \varphi^{\prime \prime}$ in decreasing on $\left.]-\infty, 0\right]$ and increasing on $[0,+\infty[$ and satisfies,

$$
\forall x \in \mathbb{R}, \quad \varphi^{\prime \prime}(x) \geqslant \varphi^{\prime \prime}(0)=\lambda>0 \text { and } \lim _{|x| \rightarrow \infty} \varphi^{\prime \prime}(x)=\infty .
$$

Assume also that there exists $A>1$ such that for $|x| \geqslant C$ for some $C>0$,

$$
A \varphi(x) \leq x \varphi^{\prime}(x) .
$$


Then there exists $C>0$ such that for all smooth enough functions $g$,

$$
\text { Ent }_{\mu_{\varphi}}\left(e^{g}\right) \leq \int H_{\varphi}\left(g^{\prime}\right) e^{g} d \mu_{\varphi}
$$

where

$$
H_{\varphi}(x)=\left\{\begin{array}{rrrr}
\frac{2 A}{A-1} \varphi^{*}\left(\frac{x}{2}\right), & \text { if } & |x|>C \\
\frac{1}{2 \lambda} x^{2}, & \text { if } & |x| \leq C .
\end{array}\right.
$$

The proof of this theorem is a straightforward application of the following lemma:

Lemma 3.2 Assume that $\varphi$ satisfies conditions of Theorem 3.1, then we get

$$
\forall x, y \in \mathbb{R}, \quad x y-\varphi^{*}\left(\varphi^{\prime}(x)\right)+\varphi^{*}\left(\varphi^{\prime}(x)-y\right) \leq H_{\varphi}(y) .
$$

\section{Proof}

$\triangleleft$ We know that for all $x \in \mathbb{R}^{n}, x=\varphi^{*^{\prime}}\left(\varphi^{\prime}(x)\right)$, and the convexity of $\varphi^{*}$ yields

$$
x y-\varphi^{*}\left(\varphi^{\prime}(x)\right)+\varphi^{*}\left(\varphi^{\prime}(x)-y\right) \leq y\left(\varphi^{*^{\prime}}\left(\varphi^{\prime}(x)\right)-\varphi^{*^{\prime}}\left(\varphi^{\prime}(x)-y\right)\right) .
$$

Let $y \in \mathbb{R}$ be fixed and notes $\psi_{y}(x)=\varphi^{*^{\prime}}(x+y)-\varphi^{*^{\prime}}(x)$. The function $\varphi$ is convex, so one gets for all $x \in \mathbb{R}, \varphi^{*^{\prime \prime}}\left(\varphi^{\prime}(x)\right) \varphi^{\prime \prime}(x)=1$, and the maximum of $\psi_{y}(x)$ is reached on $x_{0} \in \mathbb{R}$ which satisfies the condition $\varphi^{*^{\prime \prime}}\left(x_{0}\right)=\varphi^{*^{\prime \prime}}\left(x_{0}+y\right)$. Since $\varphi$ is even, $\varphi^{\prime \prime}$ is decreasing on $\left.]-\infty, 0\right]$ and increasing on ] $-\infty, 0]$ one get that $x_{0}+y=-x_{0}$. Then one obtains

$$
\begin{gathered}
\forall y \in \mathbb{R}, \quad \forall x \in \mathbb{R}, \quad \varphi^{*^{\prime}}(x+y)-\varphi^{*^{\prime}}(x) \leq 2 \varphi^{*^{\prime}}\left(\frac{y}{2}\right), \\
\forall y \in \mathbb{R}, \quad \forall x \in \mathbb{R}, \quad x y-\varphi^{*}\left(\varphi^{\prime}(x)\right)+\varphi^{*}\left(\varphi^{\prime}(x)-y\right) \leq y 2 \varphi^{*^{\prime}}\left(\frac{y}{2}\right) .
\end{gathered}
$$

By (19) one gets

$$
\forall|y| \geqslant C, \quad \forall x \in \mathbb{R}, \quad x y-\varphi^{*}\left(\varphi^{\prime}(x)\right)+\varphi^{*}\left(\varphi^{\prime}(x)-y\right) \leq \frac{2 A}{A-1} \varphi^{*}\left(\frac{y}{2}\right) .
$$

A Taylor's formula then leads to

$$
\forall x, y \in \mathbb{R}, \quad x y-\varphi^{*}\left(\varphi^{\prime}(x)\right)+\varphi^{*}\left(\varphi^{\prime}(x)-y\right) \leq \frac{y^{2}}{2} \varphi^{*^{\prime \prime}}\left(\varphi^{\prime}(x)-\theta y\right) \leq \frac{y^{2}}{2 \lambda},
$$

for some $\theta \in(0,1)$, and one gets (24). $\triangleright$

Remark 3.3 - The last theorem improved the classical consequence of Bakry-Émery criterion for the logarithmic Sobolev inequality. In fact when a probability measure is more log-concave than the Gaussian measure, we obtain a modified logarithmic Sobolev inequality sharper than the classical inequality of Gross. Using a such inequality then one obtains concentration inequality which is more adapted to the probability measure studied.

- Theorem 3.1 is more precise than Corollary 2.0 proved by Bobkov, Ledoux and Zegarlinski in BL00, BZ05]. The particularity of the function $H_{\varphi}$ defined on (21) is its behaviour around the origin. One can obtain easily that if a probability measure satisfies inequality (20) then it satisfies a Poincaré inequality with constant $1 / \lambda$.

- Note also that this method can not be applied for measures with a concentration between $e^{-|x|}$ and $e^{-x^{2}}$ described in [GGM05, GGM07]. In particular Lemma 3.9 is false in this case. 
- Note finally that the condition (19) is a technical condition, satisfied for a large class of functions.

A natural application of Theorem 3.1 is a concentration inequality in the spirit of Talagrand, see [Tal95].

Corollary 3.4 Assume that $\varphi$ satisfies conditions of Theorem 3.1 and there exists $B>1$ such that for $|x|$ large enough,

$$
x \varphi^{\prime}(x) \leq B \varphi(x) .
$$

Then there exists constants $C_{1}, C_{2}, C_{3} \geqslant 0$, independent of $n$ such that: if $F$ is a function on $\mathbb{R}^{n}$ such that $\forall i,\left\|\partial_{i} F\right\|_{\infty} \leq 1$, then we get for $\lambda \geq 0$,

$$
\mu^{\otimes n}\left(\left|F-\mu^{\otimes n}(F)\right| \geqslant \lambda\right) \leq \begin{cases}2 \exp \left(-n C_{1} \Phi\left(C_{2} \frac{\lambda}{n}\right)\right) & \text { if } \lambda>n C_{3}, \\ 2 \exp \left(-C_{1} \frac{\lambda^{2}}{n}\right) & \text { if } 0 \leq \lambda \leq n C_{3} .\end{cases}
$$

\section{Proof}

$\triangleleft$ Using the additional hypothesis (25), the proof of (26) is the same as for Proposition 3.2 of GGM07.

A $n$-dimensional version of (20) is also available.

Proposition 3.5 Let $\Phi$ be a $\mathcal{C}^{2}$, strictly convex and even function on $\mathbb{R}^{n}$ and satisfying (7) and (8). Assume also that $\Phi \geqslant 0$ and $\Phi(0)=0$ (it implies that 0 is the unique minimum of $\Phi$ ),

$$
\lim _{\alpha \rightarrow 0, \alpha \in[0,1]} \sup _{x \in \mathbb{R}^{n}}\left\{(1-\alpha) \frac{\Phi^{*}\left(\frac{x}{1-\alpha}\right)}{\Phi^{*}(x)}\right\}=1,
$$

and also that there exists $A>0$ such that

$$
\forall x \in \mathbb{R}^{n}, \quad x \cdot \nabla \Phi(x) \leq(A+1) \Phi(x) .
$$

Then there exist $C_{1}, C_{2}, C_{3} \geqslant 0$ such that for all smooth enough functions $g$ such that $\int e^{g} d \mu_{\Phi}=1$ we get

$$
\operatorname{Ent}_{\mu_{\Phi}}\left(e^{g}\right) \leq C_{1} \int \Phi^{*}\left(C_{2} \nabla g\right) e^{g} d \mu_{\Phi}+C_{3} .
$$

\section{Proof}

$\triangleleft$ Let apply Theorem 2.1 with $\varphi=\Phi+\log Z_{\Phi}$, one has

$$
\text { Ent }_{\mu_{\Phi}}\left(e^{g}\right) \leq \int\left\{x \cdot \nabla g(x)-\Phi^{*}(\nabla \Phi(x))+\Phi^{*}(\nabla \Phi(x)-\nabla g(x))\right\} e^{g} d \mu_{\Phi} .
$$

The convexity of $\Phi^{*}$ implies, for all $\alpha \in[0,1[$,

$$
\forall x \in \mathbb{R}^{n}, \quad \Phi^{*}(\nabla \Phi(x)-\nabla g(x)) \leq(1-\alpha) \Phi^{*}\left(\frac{\nabla \Phi(x)}{1-\alpha}\right)+\alpha \Phi^{*}\left(\frac{-\nabla g(x)}{\alpha}\right) .
$$

Recall that $\Phi^{*}$ is also an even function. Young's inequality implies that

$$
\forall x \in \mathbb{R}^{n}, \quad x \cdot \frac{\nabla g(x)}{\alpha} \leq \Phi(x)+\Phi^{*}\left(\frac{\nabla g(x)}{\alpha}\right) .
$$


Using (30) and (31) we get

$$
\begin{aligned}
\text { Ent }_{\mu_{\Phi}}\left(e^{g}\right) \leq 2 \alpha \int \Phi^{*}\left(\frac{\nabla g}{\alpha}\right) e^{g} d \mu_{\Phi}+\alpha \int \Phi(x) e^{g} d \mu_{\Phi}+ \\
\qquad\left((1-\alpha) \Phi^{*}\left(\frac{\nabla \Phi(x)}{1-\alpha}\right)-\Phi^{*}(\nabla \Phi(x))\right) e^{g} d \mu_{\Phi} .
\end{aligned}
$$

We have $\Phi^{*}(\nabla \Phi(x))=x \cdot \nabla \Phi(x)-\Phi(x)$, then inequality (28) implies that $\Phi^{*}(\nabla \Phi(x)) \leq A \Phi(x)$. Because of $\Phi(0)=0$ one has $\Phi^{*} \geqslant 0$, so that

$$
\text { Ent }_{\mu_{\Phi}}\left(e^{g}\right) \leq \alpha \int \Phi^{*}\left(\frac{\nabla g}{\alpha}\right) e^{g} d \mu_{\Phi}+\alpha \int \Phi^{*}\left(\frac{\nabla g}{\alpha}\right) e^{g} d \mu_{\Phi}+(\alpha+A|\psi(\alpha)-1|) \int \Phi e^{g} d \mu_{\Phi},
$$

where

$$
\psi(\alpha)=\sup _{x \in \mathbb{R}^{n}}\left\{(1-\alpha) \frac{\Phi^{*}\left(\frac{x}{1-\alpha}\right)}{\Phi^{*}(x)}\right\} .
$$

Let $\lambda>0$, recall that $\int e^{g} d \mu_{\Phi}=1$ then yields

$$
\int \Phi e^{g} d \mu_{\Phi} \leq \lambda\left(\operatorname{Ent}_{\mu_{\Phi}}\left(e^{g}\right)+\log \int e^{\Phi / \lambda} d \mu_{\Phi}\right) .
$$

One has $\lim _{\lambda \rightarrow \infty} \log \int e^{\Phi / \lambda} d \mu_{\Phi}=0$. Let then let now choose $\lambda$ large enough so that $\log \int e^{\Phi / \lambda} d \mu_{\Phi} \leq 1$. Using the property (27), taking $\alpha$ such that $(\alpha+A|\psi(\alpha)-1|) \lambda \leq 1 / 2$ implies

$$
\text { Ent }_{\mu_{\Phi}}\left(e^{g}\right) \leq 2 \alpha \int \Phi^{*}\left(\frac{\nabla g}{\alpha}\right) e^{g} d \mu_{\Phi}+\frac{1}{2}\left(\mathbf{E n t}_{\mu_{\Phi}}\left(e^{g}\right)+1\right)
$$

which gives

$$
\operatorname{Ent}_{\mu_{\Phi}}\left(e^{g}\right) \leq 4 \alpha \int \Phi^{*}\left(\frac{\nabla g}{\alpha}\right) e^{g} d \mu_{\Phi}+1 / 2 .
$$

$\triangleright$

The main difference between the inequality obtained and the modified logarithmic inequality (20) is that we do not have equality if $f=1$. Then (29) is called a no tight inequality and it is more difficult to obtain.

\subsection{Application to Euclidean logarithmic Sobolev inequality}

Theorem 3.6 Assume that the function $\varphi$ satisfies conditions of Theorem 2.1. Then for all $\lambda>0$ and for all smooth enough functions $g$ on $\mathbb{R}^{n}$,

$$
\mathbf{E n t}_{d x}\left(e^{g}\right) \leq-n \log (\lambda e) \int e^{g} d x+\int \varphi^{*}(-\lambda \nabla g) e^{g} d x
$$

This inequality is optimal in the sense that if $g=-\varphi(x-\bar{x})$ with $\bar{x} \in \mathbb{R}^{n}$ and $\lambda=1$ we get an equality.

\section{Proof}

$\triangleleft$ Integrating by parts in the second term of (9) yields for all $g$ smooth enough

$$
\int x \cdot \nabla g(x) e^{g(x)} d \mu_{\varphi}(x)=\int(-n+x \cdot \nabla \varphi(x)) e^{g(x)} d \mu_{\varphi}(x) .
$$


Then using the equality $\varphi^{*}(\nabla \varphi)=x \cdot \nabla \varphi(x)-\varphi(x)$ we get for all smooth enough functions $g$,

$$
\text { Ent }_{\mu_{\varphi}}\left(e^{g}\right) \leq \int\left(-n+\varphi+\varphi^{*}(\nabla \varphi-\nabla g)\right) e^{g} d \mu_{\varphi},
$$

Let now take $g=f+\varphi$ to obtain

$$
\operatorname{Ent}_{d x}\left(e^{f}\right) \leq \int\left(-n+\varphi^{*}(-\nabla g)\right) e^{g} d x
$$

Finally, let $\lambda>0$ and take $f(x)=g(\lambda x)$, we get then

$$
\mathbf{E n t}_{d x}\left(e^{g}\right) \leq-n \log (\lambda e) \int e^{g} d x+\int \varphi^{*}(-\lambda \nabla g) e^{g} d x,
$$

which proves (33).

If now $g=-\varphi(x-\bar{x})$ with $\bar{x} \in \mathbb{R}^{n}$ an easy computation proves that if $\lambda=1$ the equality holds. $\triangleright$

In the inequality (33), there exists an optimal $\lambda_{0}>0$ and when $C$ is homogeneous, we can improve the last result. We find an inequality called Euclidean logarithmic Sobolev inequality which is explained on the next corollary.

Corollary 3.7 Let $C$ be a strictly convex function on $\mathbb{R}^{n}$ satisfying condition of Theorem 2.1 and assume that $C$ is $q$-homogeneous for some $q>1$,

$$
\forall \lambda \geqslant 0 \quad \text { and } \quad \forall x \in \mathbb{R}^{n}, \quad C(\lambda x)=\lambda^{q} C(x) .
$$

Then for all smooth enough functions $g$ in $\mathbb{R}^{n}$ we get

$$
\operatorname{Ent}_{d x}\left(e^{g}\right) \leq \frac{n}{p} \int e^{g} d x \log \left(\frac{p}{n e^{p-1} \mathcal{L}^{p / n}} \frac{\int C^{*}(-\nabla g) e^{g} d x}{\int e^{g} d x}\right),
$$

where $\mathcal{L}=\int e^{-C} d x$ and $1 / p+1 / q=1$.

\section{Proof}

$\triangleleft$ Let apply Theorem 3.6 with $\varphi=C+\log \mathcal{L}$. Then $\varphi$ satisfies conditions of Theorem 3.6 and we get then

$$
\operatorname{Ent}_{d x}\left(e^{g}\right) \leq-n \log \left(\lambda e \mathcal{L}^{1 / n}\right) \int e^{g} d x+\int C^{*}(-\lambda \nabla g) e^{g} d x .
$$

Due to the fact that $C$ is $q$-homogeneous an easy computation proves that $C^{*}$ is $p$-homogeneous where $1 / p+1 / q=1$. An optimization over $\lambda>0$ gives inequality (34). $\triangleright$

Remark 3.8 Inequality (34) is useful to prove regularity properties as hypercontractivity for nonlinear diffusion as the p-Laplacian, see [DPDG04]. The function $C$ is then adapted to the nonlinear diffusion studied.

Inequality (34) is called Euclidean logarithmic Sobolev inequality and computations of this section is the generalization of the work of Carlen in Car91. This inequality with $p=2$, appears in the work of Weissler in [Wei78]. It was discussed and extended to this last version in many articles see Bec99, DPD03, Gen03, AGK04.

Remark 3.9 As explained in the introduction, computation used in Corollary 3.7 clearly proves that inequality (34) is equivalent to inequality (33). Agueh, Ghoussoub and Kang, in [AGK04], used Monge-Kantorovich theory for mass transport to prove inequalities (33) and (34). Their approach gives another way to establish Theorem 2.1.

Note finally that inequality (34) is optimal, extremal functions are given by $g(x)=-b C(x-\bar{x})$, with $\bar{x} \in \mathbb{R}^{n}$ and $b>0$. If they are only ones is still an open question.

Acknowledgments: I would like to warmly thank referee for pointed out errors in the first version. 


\section{References}

$\left[\mathrm{ABC}^{+} 00\right]$ C. Ané, S. Blachère, D. Chafaï, P. Fougères, I. Gentil, F. Malrieu, C. Roberto, and G. Scheffer. Sur les inégalités de Sobolev logarithmiques, volume 10 of Panoramas et Synthèses. Société Mathématique de France, Paris, 2000.

[AGK04] M. Agueh, N. Ghoussoub, and X. Kang. Geometric inequalities via a general comparison principle for interacting gases. Geom. Funct. Anal., 14(1):215-244, 2004.

[BÉ85] D. Bakry and M. Émery. Diffusions hypercontractives. In Séminaire de probabilités, XIX, 1983/84, volume 1123 of Lecture Notes in Math., pages 177-206. Springer, 1985.

[Bec99] William Beckner. Geometric asymptotics and the logarithmic Sobolev inequality. Forum Math., 11(1):105-137, 1999.

[BGL01] S. Bobkov, I. Gentil, and M. Ledoux. Hypercontractivity of Hamilton-Jacobi equations. J. Math. Pu. Appli., 80(7):669-696, 2001.

[BL00] S. G. Bobkov and M. Ledoux. From Brunn-Minkowski to Brascamp-Lieb and to logarithmic Sobolev inequalities. Geom. Funct. Anal., 10(5):1028-1052, 2000.

[BZ05] S.G. Bobkov and B. Zegarlinski. Entropy bounds and isoperimetry. Mem. Am. Math. Soc., 829:69 p., 2005.

[Car91] E. A. Carlen. Superadditivity of Fisher's information and logarithmic Sobolev inequalities. J. Funct. Anal., 101(1):194-211, 1991.

[CEGH04] D. Cordero-Erausquin, W. Gangbo, and C. Houdré. Inequalities for generalized entropy and optimal transportation. In Recent advances in the theory and applications of mass transport, volume 353 of Contemp. Math., pages 73-94. Amer. Math. Soc., Providence, RI, 2004.

[DPD03] M. Del Pino and J. Dolbeault. The optimal Euclidean $L^{p}$-Sobolev logarithmic inequality. J. Funct. Anal., 197(1):151-161, 2003.

[DPDG04] M. Del Pino, J. Dolbeault, and I. Gentil. Nonlinear diffusions, hypercontractivity and the optimal $L^{p}$-Euclidean logarithmic Sobolev inequality. J. Math. Anal. Appl., 293(2):375388,2004 .

[Gen03] I. Gentil. The general optimal $L^{p}$-Euclidean logarithmic Sobolev inequality by HamiltonJacobi equations. J. Funct. Anal., 202(2):591-599, 2003.

[GGM05] I. Gentil, A. Guillin, and L. Miclo. Modified logarithmic Sobolev inequalities and transportation inequalities. Probab. Theory Related Fields, 133(3):409-436, 2005.

[GGM07] I. Gentil, A. Guillin, and L. Miclo. Logarithmic sobolev inequalities in curvature null. Rev. Mat. Iberoamericana, 23(1):237-260, 2007.

[Gro75] L. Gross. Logarithmic Sobolev inequalities. Amer. J. Math., 97(4):1061-1083, 1975.

[Gup80] S. D. Gupta. Brunn-Minkowski inequality and its aftermath. J. Multivariate Anal., 10:296-318, 1980.

[Mau04] B. Maurey. Inégalité de Brunn-Minkowski-Lusternik, et autres inégalités géométriques et fonctionnelles. Séminaire Bourbaki, 928, 2003/04.

[OV00] F. Otto and C. Villani. Generalization of an inequality by Talagrand, and links with the logarithmic Sobolev inequality. J. Funct. Anal., 173(2):361-400, 2000. 
[Tal95] M. Talagrand. Concentration of measure and isoperimetric inequalities in product spaces. Inst. Hautes Études Sci. Publ. Math., (81):73-205, 1995.

[Wei78] F. B. Weissler. Logarithmic Sobolev inequalities for the heat-diffusion semigroup. Trans. Am. Math. Soc., 237:255-269, 1978. 